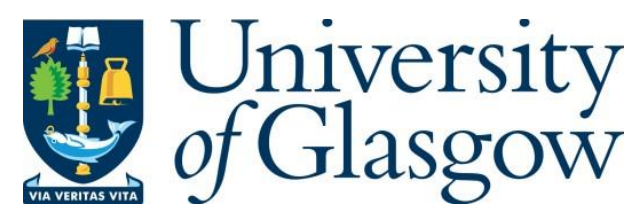

Freeman, E., Williamson, J. R., Kourtelos, P. and Brewster, S. (2018) Levitating Particle Displays with Interactive Voxels. In: 7th ACM International Symposium on Pervasive Displays (PerDis '18), Munich, Germany, 06-08 Jun 2018, p. 15. ISBN 9781450357654.

There may be differences between this version and the published version. You are advised to consult the publisher's version if you wish to cite from it.

http://eprints.gla.ac.uk/161698/

Deposited on: 3 May 2018

Enlighten - Research publications by members of the University of Glasgow http://eprints.gla.ac.uk 


\title{
Levitating Particle Displays with Interactive Voxels
}

\author{
Euan Freeman, Julie R. Williamson, Praxitelis Kourtelos, and Stephen Brewster \\ Glasgow Interactive Systems Section \\ School of Computing Science \\ University of Glasgow, Scotland \\ \{first.last\}@glasgow.ac.uk
}

\begin{abstract}
Levitating objects can be used as the primitives in a new type of display. We present levitating particle displays and show how research into object levitation is enabling a new way of presenting and interacting with information. We identify novel properties of levitating particle displays and give examples of the interaction techniques and applications they allow. We then discuss design challenges for these displays, potential solutions, and promising areas for future research.
\end{abstract}

\section{ACM Classification Keywords}

H.5.2. User Interfaces: Interaction styles.

\section{Author Keywords}

Acoustic levitation; levitation; particle displays; voxels.

\section{INTRODUCTION}

Object levitation enables new types of display, where content is created from physical "particles" in air instead of pixels on a surface. Several technologies have been developed to allow objects to be levitated and manipulated in mid-air, with particles ranging from small polystyrene beads [21] to large spherical magnets [12] to drones [8]. Such displays create new opportunities for interaction: users can see content from many angles and can look through the display; they can 'reach in' and interact in the empty spaces around and between particles; the physical properties of the particles and their effects on their surroundings can be meaningful in the interaction; and users can leverage their understanding of the physical world to better understand the content they are interacting with.

These new interaction possibilities also come with challenges. Users cannot always manipulate levitating particles in the same way they might manipulate pixels on a screen due to levitation constraints, so new interaction techniques are required. The display elements are physical objects, so updating the display is not straightforward and special rendering techniques are needed. Simply getting the particles into the air in the first place may require some effort from the user, so this becomes

This is the author's copy of this paper. This will appear in the proceedings of the 7th ACM International Symposium on Pervasive Displays.

https://doi.org/10.1145/3205873.3205878 part of the interaction and solutions are needed to support this. Such challenges can be overcome and we think this is worthwhile because of the potential of these displays.

In this paper, we discuss recent advances in levitation research and how these are being used to enable a novel display medium. We identify several ways of using levitating particles as voxels (pixels in a 3D volume) and discuss interactions that take advantage of their unique capabilities. We also look at how users can manipulate content shown using these displays and the interaction challenges that need to be addressed to allow this. By putting forth this design space, we hope to encourage others to come up with novel interactions based on levitation.

\section{BACKGROUND}

Levitation allows physical "particles" to be positioned and controlled in mid-air. These particles can be many types of object/material (even liquid [26]). We envision a new type of display where each particle is a "voxel" (a pixel in a volumetric display) that can be used as a display primitive in mid-air. The content of such a display can be changed by manipulating the appearance and position of each voxel, or by augmenting it with auditory and haptic information.

Several approaches have been developed for levitating objects and manipulating them in mid-air. ZeroN [12] (see Figure 1) used magnetic levitation to levitate a single spherical object. The object could be repositioned by manipulating the magnetic field in the display volume. While limited to only one particle, this system supported rich visual output by projecting onto the surface of the sphere and onto the flat surface beneath it. As shown in Figure 1, other objects could also be introduced into the display volume to enhance the levitation and users could 'reach in' to touch and move the levitating particle.
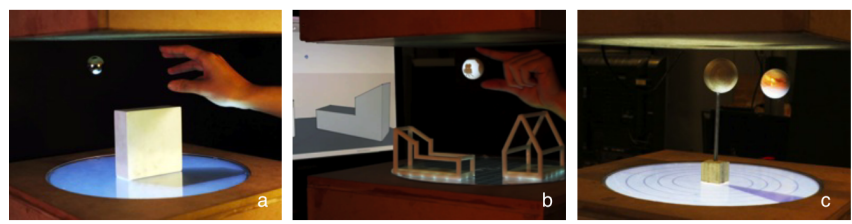

Figure 1. ZeroN [12] levitated a magnetic sphere, which was also used as a projection surface.

Aerial Tunes [1] and Floatio [28] used airflow to levitate and move spherical objects in mid-air. As shown in Figure 2, this method uses a dedicated air jet for each particle. Particle height depends on the amount of airflow and the actuated air jet allows limited control over object position. Users can grasp 
and reposition objects directly when combined with a handtracker (as in [28]), although interaction needs consideration to not block the direction of airflow.

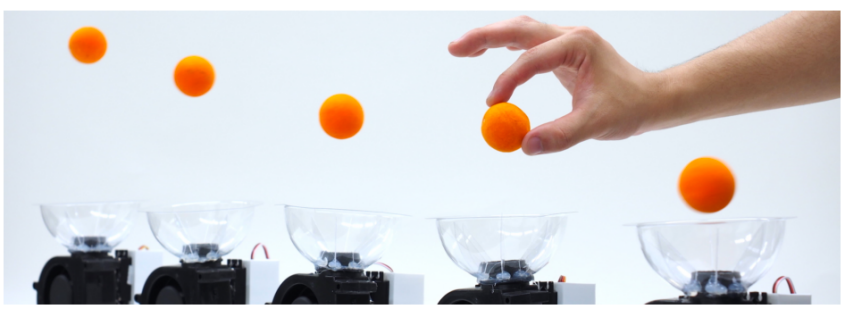

Figure 2. Floatio [28] used airflow to levitate spherical objects. Hand tracking enabled interaction with the particles.

Pixie Dust [19] used acoustics to levitate objects: arrays of ultrasound transducers created an inaudible sound field that 'trapped' the objects. This supported larger numbers of smaller objects $(\varnothing 1-5 \mathrm{~mm})$ positioned close together (at least $4.5 \mathrm{~mm}$ apart). They used objects to form 2D graphics in a flat plane, and also projected graphics onto the objects. In this system, the flat and dense layout was used like a 'screen' in mid-air, with each particle acting as a pixel for display.

Acoustic levitation allows particles to be moved in 3D, through changes in the acoustic field from the ultrasound transducer arrays. We omit technical details here but Andrade et al. [2] is a good resource for better understanding this method. LeviPath [21] (Figure 3, Left) used 3D object movement, with an approach that can be used to move more than one object independently. Point-and-Shake [7] (Figure 3, Right) used such levitating particle movement to create interactive voxels.
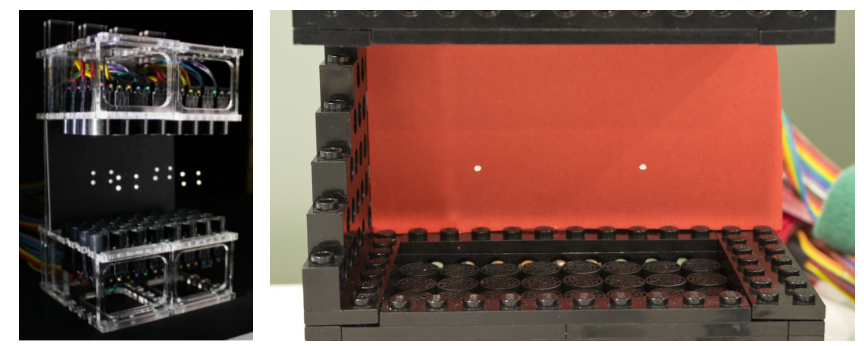

Figure 3. Left: LeviPath [21] allowed independent particle movement in 3D. Right: Point-and-Shake [7] used animated levitating particles to create interactive voxels with real-time feedback.

The most common acoustic levitation method utilises standing waves. These are stationary sound waves between two arrays of ultrasound transducers (as in Figure 3) or between one array and a surface that reflects sound. When using this method, the largest particle diameter is approx. $0.5 \lambda$; this is $4.5 \mathrm{~mm}$ for the systems we discussed here, which use $40 \mathrm{kHz}$ ultrasound.

Alternative acoustic levitation methods have been developed in recent years. Marzo et al. [15] developed holographic acoustic elements, which were capable of levitating objects above a single array of ultrasound transducers. Recent work used a similar approach to levitate larger objects of $1.17 \lambda$ [14].

Ultrasound arrays use digital phase delays to shape and focus the sound. This can also be done with acoustic metamaterials [17], which are physical 'bricks' that can be used to introduce analogue delays with a higher resolution. Like acoustic holographic elements, this method also allows singlesided levitation. Such approaches could be used to allow a full hemisphere of visibility with acoustic levitation and users would have more space to interact in without disturbing the levitation forces.

Acoustic systems are not limited to just simple materials. TastyFloats [26] levitated food and liquid particles. Presenting 'meals' in this way altered the perception of taste and demonstrated a novel way of including taste in interaction. Acoustic levitation can also be enhanced with other objects placed around or amongst the levitating particles [23], so long as they do not reflect sound. Other research has used coloured particles [22, 23] and particles physically joined by string [22] to further enhance the display capability.

Drones have also been explored as a type of 'pixel' that users can interact with in mid-air. BitDrones [8] was a framework for drone-based displays, with varying display and interaction capabilities. Flyables [11] considered interactions with drones, allowing users to touch and move them in mid-air. These devices are much bigger than the other voxels considered so far, but can allow a much larger space to be used for display. Users could step into such displays and move around the voxels, something not possible with other technologies.

\section{Summary: Levitating Particle Displays}

Several approaches have been discussed for creating levitating particle displays. Each method has strengths and weaknesses that will affect their suitability for different user interfaces. For example, magnetic levitation allows just a single particle that users can physically grasp and reposition [12], whilst acoustic levitation allows several particles but users must interact with from a distance [7]. All share the properties that make levitating particle displays novel, however. We discuss these properties later in the paper.

Levitating particles are the "voxels" in this new type of display. The most basic use of these voxels is to represent information through their relative position: for example, showing data with Floating Charts [22] or forming shapes in Pixie Dust [19]. Particles become more expressive when moved over time: for example, 'shaking' when a user interacts with them [7] or mimicking the movements a button makes when pressed [5].

Voxels can be augmented by projecting onto them $[12,19]$ or surrounding surfaces [12]. They can even be screens themselves [8]. They can be enriched through use of colour [22, $23]$, by objects added to the display volume [12, 22, 23] and through the use of auditory or haptic effects.

\section{INTERACTING WITH LEVITATING PARTICLE DISPLAYS}

Levitating particle displays have unique properties that create new opportunities for interaction. Their voxels are levitated in mid-air, allowing users to look through the display and its content and letting them see it from many angles. In some cases, users can directly 'reach inside' the display and interact with the voxels or in the empty spaces between them. Levitating particles can be used to represent information through their 
spatial and temporal characteristics, but the physical properties of the particles themselves can be meaningful within the displayed content: for example, their appearance, material and tactile characteristics. These may also be enhanced digitally: for example, projecting onto the particles. Finally, the effects of levitating particles on their surroundings can also be meaningful within an interactive application. We now discuss each of these properties in turn and use them to highlight their potential for novel interactions and applications.

\section{Voxels in an invisible volume}

Levitating particles are the voxels in the display and their appearance within the display volume is used to represent information. Unlike typical screens where display elements are contiguous, levitating particles need to be spaced apart. This is often out of necessity; for example, acoustically levitated particles need to be at least $0.5 \lambda$ apart, or further to allow space for animated movements [7]. As a result, users can see through the display volume and the content within.

The invisible volume presents opportunities for novel applications. Multiple users can view the content but also see each other through the display, without a physical barrier that impedes collaboration [3]. This property could be especially useful for education applications, as groups of students could experience the same content together whilst gathered around the display. As two examples of this, consider the use of levitating particles to simulate planetary orbits around a star and to represent molecular structures. In these applications, the invisible volume means only the important content is visible (i.e., the planets and the molecules). Users can see through the empty space to focus on the voxel structure.

Levitating particles could also be used with other types of display, with the second display rendering the content that 'fills in' the empty space. Marzo et al. [16] discussed several ways of combining levitation with other displays. The Ultra-Tangibles [13] system demonstrates this idea as well. They augmented a tablet computer with ultrasound transducers facing in towards the screen, so that polystyrene balls could be moved across the surface. This was used to create several versions of a Pong game, where the 'particle' was a tangible instance of the ball in the game, with the tablet surface used to render the players and other visual effects. Combining levitation with other displays enables designers to enhance applications with physical elements.

\section{Reaching into the display}

The empty space between voxels means users can reach into the display volume and interact in new ways. Hands-on interaction with acoustically-levitated particles is not ideal, because the hands would disrupt the acoustic field, but other levitation approaches do allow users to physically grasp and manipulate the objects. ZeroN [12] demonstrated interactions where users could alter the system state by grasping and moving objects around the interaction volume: for example, repositioning a virtual light source to see the effects on the scene.

Designers can also enhance the space surrounding the levitating particles by adding new objects or materials. ZeroN [12] used physical items as props to augment the planet application described previously (Figure 1, right) and Floating Charts [22] used materials like string and felt to enhance the appearance of their levitating datasets. Levitating particle displays have the novel ability of allowing physical objects to be placed directly inside the display volume. This could lead to new applications where users use tangible objects to manipulate content.

\section{Expressive voxels}

The appearance and behaviour of particles can be manipulated in many ways to represent information. The simplest way of encoding information using a particle is its position in the display, relative to other voxels. This is how Floating Charts [22] presented numerical data as a type of physical visualisation [10]. Unlike other 'physicalisations' with fixed physical form, however, the use of a levitating particle display means the form can be dynamically changed over time. This means particles can represent information temporally as well as spatially; in the context of data presentation, a dynamic physical data representation such as this could enable new ways of exploring complex data [24, 25].

Spatial changes have also been used to 'animate' particles as a means of giving feedback. In recent work [7], we developed a mid-air gesture technique for selecting particles, with particles 'shaking' when users pointed at them. For more complex interactions, the movement speed was altered to differentiate system states. This was a simple but effective way of using particle behaviour to represent system state as feedback.

Several strategies have been used to alter the appearance of levitating particles. Pixie Dust [19] and ZeroN [12] projected onto the particles, whilst JOLED [23] rotated particles with different coloured sides. These techniques allow simple colour change but with sufficiently large particles [12], projection can also allow quite detailed visuals. BitDrones [8] levitated small screens, enabling even more expressive output.

The particle objects themselves could be varied in meaningful ways, so that their material properties represent information to users. For example, one application of ZeroN [12] was to allow users to explore planetary orbits around a star. Users could reposition the planet to see the effect of distance on the orbit. This application could be enhanced through the use of different levitating particles: e.g., particles with different size or weight to explore the effects of these properties on orbit. As users manually replace these levitating objects, they see and feel the differences between the particles and this feeds back into their understanding of the systems being simulated.

\section{Voxels that affect their surroundings}

Earlier in this section we discussed how the space surrounding the levitating particles can be enhanced with other objects or displays, but the particles themselves can also affect the appearance or behaviour of their surroundings, leading to new types of interaction. Aerial Tunes [1] used levitating particles to represent music properties within a soundscape; when users grasped and repositioned the objects, the soundscape was updated to reflect the changes. This could create the impression that these objects have special audible qualities, because users attribute the sounds to the levitating objects, rather than the result of their actions (a form of ventriloquism [18]). 
ZeroN [12] demonstrated manipulations with a visible effect on surroundings: as users moved the levitating 'light source', the change in position affected shadows cast by other objects in the display volume (Figure 1, left). This interaction took advantage of many unique properties of levitating particle displays and shows how the surrounding space can be used as an integral part of the display. Although similar interactions can be presented using screens and pointers, levitating particles could allow users to form a better understanding, or execute more precise control, through direct manipulation of the content and its environment.

\section{Colocated audio and haptic feedback}

All of the acoustic levitation systems discussed earlier use $40 \mathrm{kHz}$ ultrasound, with one or more arrays of transducers (e.g., Figure 3, Right shows one of our devices with two $8 \times 4$ arrays). Similar hardware and acoustic principles are used to create ultrasound haptic feedback [4, 9], allowing users to experience vibration on their skin without touching anything. Likewise, the same hardware can also be used for directional audio, presenting sound that is only audible in certain regions [20].

It is not currently possible to combine acoustic levitation with ultrasound haptics or directional audio, due to interference between the sound fields. However, future acoustic techniques could allow these to be combined, creating levitating particles with colocated haptic and audio feedback. Directional audio techniques (e.g., [20]) could be used to create sound effects that come directly from the display voxels, allowing more information to be presented by the display.

Magnetic levitation could also be enhanced with haptic feedback inside the display volume. FingerFlux [27] used small magnets worn on the fingertips to give haptic feedback, by manipulating the magnetic field above a screen to attract or repel the magnets. A similar approach could be used inside a particle display, with finger magnets affected by the levitation.

\section{DESIGN CHALLENGES}

Levitating particle displays enable new interactions but also present new design challenges that need to be addressed. We present three open design challenges that must be addressed to overcome the practical barriers currently preventing the more widespread use of levitating particle displays.

\section{Preparing a levitating particle display}

When using a levitating particle display for the first time, the first challenge is getting the particles to levitate in the correct position: with current hardware, a user would need to place the particles 'into' the levitation. This could be difficult without knowing how many particles need to be added and where they need to be precisely placed, especially in systems where the particles are so small. This problem does not exist with most other displays, e.g., pixels in a screen are always ready for use.

This problem could be addressed through automation (e.g., a hardware solution that 'funnels' particles into the volume so they can moved into position) or new interaction techniques (e.g., guiding users as they place particles). Giving feedback to guide users may be difficult, especially when the particles are the primary visual component of the display, but other modalities could be used to present information. For example, auditory feedback or simple visual cues from a light source could be used to guide the user to the right location [6] as they move a particle into position (e.g., using a particle holder).

Preparing the particles could become an important part of the interaction: it presents an opportunity for users to better understand the content shown by the display, so the configuration process could integrated into the application itself. For example, in the molecular structure example used earlier, placing the 'atoms' into the levitation in the correct places could be an educational part of the experience.

\section{Manipulating a levitation}

Levitating particle displays require new interaction techniques, because the voxels are more complex than simple pixels on screens. Research on interaction with such displays has been limited so far, but there are techniques that take inspiration from other types of user interface. For example, ZeroN [12] was inspired by tangible interactions and Point-and-Shake [7] adapted gestures first used with volumetric displays.

Research is needed to create a richer interaction vocabulary for levitating particle displays. Mid-air gestures have been developed for particle selection [7], but this is just the first step in interactive levitation. New techniques are needed to allow users to manipulate levitating content or to access the underlying data (e.g., with new gesture techniques). These can draw inspiration from other modalities, although this is not straightforward as they will need to be adapted for a levitating particle display. For example, input actions need to be sensitive to the constraints of the levitation device and feedback to support interaction needs to be designed with the particle display's capabilities in mind (like in [7]).

\section{Updating the display}

Levitating particle displays support independent movement of their voxels, meaning the content on the display can be changed. However, because the voxels are physical objects, display updates need to take place without the voxels colliding. Particles will need to move around each other, since they cannot overlap or move through each other. This may require novel algorithms for transitioning from one display state to another, if significant reorganisation is required. Solutions will also depend on the levitation method used. For example, acoustic levitation allows 3D positional control, but the use of sound waves means one particle cannot pass over the top of another; instead, it needs to go around it (or move both). There may also be cases where users need to add or remove particles. This may need user intervention but could use the same techniques as when configuring the display.

\section{CONCLUSION}

We presented our vision of levitating particle displays, a new way of presenting and interacting with digital information. We identified novel properties of this new type of display and the interactions they could enable. Levitating particles are situated in an invisible volume that users can see through, reach into, and use tangible props in. The particles themselves can be used in many ways to display content: through their appearance, 
behaviour, physical properties and position. Some levitation methods also allow audio and haptic effects within the display. These design possibilities allow novel interactions, but also present new challenges for designers. We discussed what we consider to be the three main ones, opening a new space for research. With this paper, we encourage others to think of new applications with levitating particle displays and techniques for successfully interacting with them.

\section{ACKNOWLEDGEMENTS}

This research is funded by the European Union Horizon 2020 research and innovation programme (\#737087: Levitate).

\section{REFERENCES}

1. Tobias Alrøe, Jonas Grann, Erik Grönvall, Marianne Graves Petersen, and Jesper L. Rasmussen. 2012. Aerial Tunes: Exploring Interaction Qualities of Mid-air Displays. In Proceedings of the 7th Nordic Conference on Human-Computer Interaction - NordiCHI '12. ACM Press, 514-523. DOI: http://dx.doi.org/10.1145/2399016.2399095

2. Marco A. B. Andrade, Nicolas Perez, and Julio C. Adamowski. 2017. Review of Progress in Acoustic Levitation. Brazilian Journal of Physics (2017), 1-24. DOI : http://dx.doi .org/10.1007/s13538-017-0552-6

3. John Bolton, Kibum Kim, and Roel Vertegaal. 2012. A comparison of competitive and cooperative task performance using spherical and flat displays. In Proceedings of the ACM 2012 conference on Computer Supported Cooperative Work - CSCW'12. ACM Press, 529-538. DOI :

http://dx.doi.org/10.1145/2145204.2145286

4. Thomas Carter, Sue Ann Seah, Benjamin Long, Bruce Drinkwater, and Sriram Subramanian. 2013.

UltraHaptics: Multi-Point Mid-Air Haptic Feedback for Touch Surfaces. In Proceedings of the 26th Symposium on User Interface Software and Technology - UIST '13. ACM Press, 505-514. D0I :

http://dx.doi.org/10.1145/2501988.2502018

5. Euan Freeman, Ross Anderson, Carl Andersson, Julie Williamson, and Stephen Brewster. 2017. Floating Widgets: Interaction with Acoustically-Levitated Widgets. In Proceedings of the 2017 ACM International Conference on Interactive Surfaces and Spaces - ISS '17 Demos. ACM Press, 417-420. DOI: http://dx.doi.org/10.1145/3132272.3132294

6. Euan Freeman, Stephen Brewster, and Vuokko Lantz. 2016. Do That, There: An Interaction Technique for Addressing In-Air Gesture Systems. In Proceedings of the 2016 CHI Conference on Human Factors in Computing Systems - CHI '16. ACM Press, 2319-2331. DOI : http://dx.doi.org/10.1145/2858036.2858308

7. Euan Freeman, Julie Williamson, Sriram Subramanian, and Stephen Brewster. 2018. Point-and-Shake: Selecting from Levitating Object Displays. In Proceedings of the 36th Annual ACM Conference on Human Factors in Computing Systems - CHI '18. ACM Press, to appear. DOI : http://dx.doi.org/10.1145/3173574.3173592
8. Antonio Gomes, Calvin Rubens, Sean Braley, and Roel Vertegaal. 2016. BitDrones: Towards Using 3D Nanocopter Displays as Interactive Self-Levitating Programmable Matter. In Proceedings of the 2016 CHI Conference on Human Factors in Computing Systems CHI'16. ACM Press, 770-780. DOI : http://dx.doi.org/10.1145/2858036.2858519

9. Takayuki Iwamoto, Mari Tatezono, and Hiroyuki Shinoda. 2008. Non-contact method for producing tactile sensation using airborne ultrasound. In Proceedings of EuroHaptics 2008. Springer, 504-513. DOI : http://dx.doi.org/10.1007/978-3-540-69057-3_64

10. Yvonne Jansen, Pierre Dragicevic, Petra Isenberg, Jason Alexander, Abhijit Karnik, Johan Kildal, Sriram Subramanian, and Kasper Hornbaek. 2015. Opportunities and Challenges for Data Physicalization. In Proceedings of the 33rd Annual ACM Conference on Human Factors in Computing Systems - CHI'15. ACM Press, 3227-3236. DOI : http://dx.doi.org/10.1145/2702123.2702180

11. Pascal Knierim, Thomas Kosch, Alexander Achberger, and Markus Funk. 2018. Flyables: Exploring 3D Interaction Spaces for Levitating Tangibles. In Proceedings of the Twelfth International Conference on Tangible, Embedded, and Embodied Interaction - TEI' 18. ACM Press, 329-336. D0I : http://dx.doi.org/10.1145/3173225.3173273

12. Jinha Lee, Rehmi Post, and Hiroshi Ishii. 2011. ZeroN: Mid-Air Tangible Interaction Enabled by Computer Controlled Magnetic Levitation. In Proceedings of the 24th annual ACM symposium on User interface software and technology - UIST' 11. ACM Press, 327-366. DOI : http://dx.doi.org/10.1145/2047196.2047239

13. Mark T. Marshall, Tom Carter, Jason Alexander, and Sriram Subramanian. 2012. Ultra-Tangibles: Creating Movable Tangible Objects on Interactive Tables. In Proceedings of the SIGCHI Conference on Human Factors in Computing Systems - CHI '12. ACM Press, 2185-2188. http://dl.acm. org/citation. cfm?id=2208370

14. Asier Marzo, Mihai Caleap, and Bruce W. Drinkwater. 2018. Acoustic Virtual Vortices with Tunable Orbital Angular Momentum for Trapping of Mie Particles. Physical Review Letters 120, 4 (2018), Article 044301. DOI : http://dx.doi .org/10.1103/PhysRevLett. 120.044301

15. Asier Marzo, Sue Ann Seah, Bruce W. Drinkwater, Deepak Ranjan Sahoo, Benjamin Long, and Sriram Subramanian. 2015. Holographic acoustic elements for manipulation of levitated objects. Nature Communications 6 (2015), Article 8661. DOI : http://dx. doi .org/10.1038/ncomms9661

16. Asier Marzo, Sriram Subramanian, and Bruce W. Drinkwater. 2017. LeviSpace: Augmenting the Space Above Displays with Levitated Particles. Proceedings of the 2017 ACM International Conference on Interactive Surfaces and Spaces (2017), 442-445. DOI : http://dx.doi.org/10.1145/3132272.3132295 
17. Gianluca Memoli, Mihai Caleap, Michihiro Asakawa, Deepak R. Sahoo, Bruce W. Drinkwater, and Sriram Subramanian. 2017. Metamaterial bricks and quantization of meta-surfaces. Nature Communications 8 (2017), Article 14608. DOI:

http://dx.doi.org/10.1038/ncomms 14608

18. Jörg Müller, Matthias Geier, Christina Dicke, and Sascha Spors. 2014. The BoomRoom: Mid-air Direct Interaction with Virtual Sound Sources. In Proceedings of the SIGCHI Conference on Human Factors in Computing Systems - CHI '14. ACM Press, 247-256. D0I : http://dx.doi.org/10.1145/2556288.2557000

19. Yoichi Ochiai, Takayuki Hoshi, and Jun Rekimoto. 2014. Pixie Dust: Graphics Generated by Levitated and Animated Objects in Computation Acoustic-Potential Field. ACM Transactions on Graphics 33, 4 (2014), Article 85. DOI : http://dx.doi.org/10.1145/2601097.2601118

20. Yoichi Ochiai, Takayuki Hoshi, and Ippei Suzuki. 2017. Holographic Whisper: Rendering Audible Sound Spots in Three-dimensional Space by Focusing Ultrasonic Waves. In Proceedings of the 2017 CHI Conference on Human Factors in Computing Systems - CHI'17. ACM Press, 4314-4325. DOI : http://dx.doi.org/10.1145/3025453.3025989

21. Themis Omirou, Asier Marzo, Sue Ann Seah, and Sriram Subramanian. 2015. LeviPath: Modular Acoustic Levitation for 3D Path Visualisations. In Proceedings of the 33rd Annual ACM Conference on Human Factors in Computing Systems - CHI'15. ACM Press, 309-312. DOI : http://dx.doi.org/10.1145/2702123.2702333

22. Themis Omirou, Asier Marzo Perez, Sriram Subramanian, and Anne Roudaut. 2016. Floating Charts: Data Plotting using Free-Floating Acoustically Levitated Representations. In 2016 IEEE Symposium on 3D User Interfaces (3DUI). IEEE, 187-190. DOI : http://dx.doi.org/10.1109/3DUI. 2016.7460051

23. Deepak Ranjan Sahoo, Takuto Nakamura, Asier Marzo, Themis Omirou, Michihiro Asakawa, and Sriram
Subramanian. 2016. JOLED: A Mid-air Display based on Electrostatic Rotation of Levitated Janus Objects. In Proceedings of the 29th Annual Symposium on User Interface Software and Technology - UIST '16. ACM Press, 437-448. DOI : http://dx.doi.org/10.1145/2984511.2984549

24. Faisal Taher, John Hardy, Abhijit Karnik, Christian Weichel, Yvonne Jansen, Kasper Hornbæk, and Jason Alexander. 2015. Exploring Interactions with Physically Dynamic Bar Charts. In Proceedings of the SIGCHI Conference on Human Factors in Computing Systems CHI '15. ACM Press, 3237-3246. DOI : http://dx.doi.org/10.1145/2702123.2702604

25. Faisal Taher, Yvonne Jansen, Jonathan Woodruff, John Hardy, Kasper Hornbæk, and Jason Alexander. 2017. Investigating the Use of a Dynamic Physical Bar Chart for Data Exploration and Presentation. IEEE

Transactions on Visualization and Computer Graphics 23, 1 (2017), 451-460. DOI:

http://dx.doi.org/10.1109/TVCG.2016.2598498

26. Chi Than Vi, Asier Marzo, Damien Ablart, Gianluca Memoli, Sriram Subramanian, Bruce Drinkwater, and Marianna Obrist. 2017. TastyFloats: A Contactless Food Delivery System. In Proceedings of the 2017 ACM International Conference on Interactive Surfaces and Spaces - ISS '17. ACM Press, 161-170. DOI: http://dx.doi.org/10.1145/3132272.3134123

27. Malte Weiss, Simon Voelker, Jan Borchers, and Chat Wacharamanotham. 2011. FingerFlux: Near-surface Haptic Feedback on Tabletops. In Proceedings of the 24th Symposium on User Interface Software and Technology UIST'11.615-620.

28. Toshiya Yui and Tomoko Hashida. 2016. Floatio: Floating Tangible User Interface Based on Animacy Perception. In Adjunct Proceedings of the 29th Annual Symposium on User Interface Software and Technology UIST'16 Adjunct. ACM Press, 43-45. DOI : http://dx.doi.org/10.1145/2984751.2985699 